\title{
STOCHASTIC METHODS FOR NONLINEAR ROTORDYNAMICS WITH UNCERTAINTIES
}

\author{
Edoardo Peradotto \\ Department of Aerospace Engineering \\ Politecnico di Torino, École Centrale de Lyon \\ Turin, 10129, Italy \\ Email: edoardoperadotto@yahoo.it \\ Loïc Salles \\ Vibration University Technology Center \\ Imperial College London \\ London, SW72AZ, United Kingdom \\ Email: I.salles@imperial.ac.uk
}

\author{
Alfonso M. Panunzio \\ Laboratoire MSSMat \\ École Centrale de Paris \\ Châtenay-Malabry, 92290, France \\ Email: alfonso.panunzio@ecp.fr
}

\author{
Christoph Schwingshackl \\ Vibration University Technology Center \\ Imperial College London \\ London, SW72AZ, United Kingdom \\ Email: c.schwingshackl@imperial.ac.uk
}

\section{ABSTRACT}

The calculated dynamic response of an excited rotating system can be significantly affected by uncertainties in its mechanical properties, such as mass, stiffness, geometrical imperfections, or loadings. For this reason, it is essential to understand and quantify the influence of uncertain parameters on the predicted rotor response.

This paper aims to optimize the propagation of random input uncertainties for a rotordynamic problem and assess their influence on the dynamic behaviour of an unbalanced rotor. The Harmonic balance method (HBM) and a non-intrusive Polynomial Chaos Expansion (PCE) are used to evaluate the stochastic response of a finite element rotor. The proposed stochastic approach is based on a numerical quadrature calculation of integrals for finding the coefficients of the PCE.

The method is initially applied to evaluate the stochastic response of a linear rotodynamic system, leading to the original concept of stochastic Campbell diagram and further extended to nonlinear rotordynamic problems, using the Asymptotic Numerical Method (ANM).

\section{NOMENCLATURE}

$\omega, \xi$ frequency,random variable

$\boldsymbol{M}, \boldsymbol{G}, \boldsymbol{K}$ mass,gyroscopic and stiffness matrices

$\Psi_{k} \quad$ Polynomial chaos basis function

$\beta_{k} \quad k$-th stochastic mode

ANM Asymptotic Numerical Method.

HBM Harmonic Balance Method.

PCE Polynomical Chaos Expansion.

\section{INTRODUCTION}

Assessing the influence of uncertainties has become a topic of great interest in engineering, especially in the robust design of high technological products. Turbomachines require mathematical models that are able to precisely describe the real behaviour of the system, considering all the possible uncertainties of input parameters that can related to uncertainties of the material properties, the tolerance in the dimensions, uncertain operating range and errors in modelling. In order to robustly design it is 
necessary to be able to quantify the propagation of the uncertainties. These uncertainties, as well as the system response, can be modelled by random variables, characterized by a some kind of probabilistic distribution. The most common method to deal with uncertainties is the Monte Carlo Simulation (MCS), a statistical sampling technique created in the 1940s [1]. The direct MCS creates a large sample of stochastic responses, in order to represent its probabilistic distribution, hence it requires a large number of repeated solutions of the same deterministic problem with different parameters.

Although very robust and flexible, MCS is affected by a slow convergence rate $\left(O\left(N^{-\frac{1}{2}}\right)\right.$, especially for large and complex systems, but convergence can be always assured [2]. This leads to expensive simulations, both in terms of time consumption and computational costs. This study proposes the use of the Polynomial Chaos Expansion (PCE), which is generally more effective than the MCS due to a substitution of uncertain parameters with a metamodel [3]. Originally proposed by Wiener [4], the main idea is to represent a stochastic process as an orthogonal base of polynomials. Ghanem and Spanos [2] first applied this method to a finite element (FE) application allowing the stochastic evaluation of large scale models. Xiu and Karniadakis [5] added non-Gaussian distributions by adapting the polynomial basis to different probabilistic distribution of the input parameters for optimal convergence. Subsequently, PCE concepts were extended to different random spaces, such as rational function series [6], partitioned random spaces [7] and sparse chaos expansions [8] which permit improvements in accuracy and the convergence of the method.

Two major numerical approaches are available to solve a stochastic problem with the PCE. The Galerkin projection [3] uses analytical expression of the stochastic matrix, which has to be explicitly implemented in the code. Didier et al. [9] used this approach to analyse the dynamic response of a rotor under uncertain parameter. As an alternative the stochastic collocation method [10] can be used, which does not require system dependent equations, and can consequently be easily adapted for different problems.

This paper focuses on the propagation of uncertainties on the vibrational response of a rotor model. The PCE has been applied to a linear and a nonlinear system, where the considered rotor is intentionally simple, in order to easily implement and validate the PCE and show its effectiveness. The uncertain system response has been applied to the Frequency Response Function $(F R F)$ and the Campbell Diagram. The study is based on the interaction of two open-source software solutions, the FE solver Code Aster [11] and the stochastic library OpenTURNS [12]. and their coupling with the help of Python.Newly developed codes call and manage the interactions between existing software and are also used to define the system equations.
The PCE is combined with the harmonic balance method (HBM) and the Asymptotic Numerical Method (ANM) [13] to evaluate the vibrational behaviour of a non-linear system with some uncertain parameters. A non-intrusive approach is used to get the stochastic response. For multivariate random spaces, the Smolyak quadrature [14] is used effectively to reduce the number of required iterations.

A numerical example based on a finite element rotor proposed by Didier [9] illustrates the method proposed in this paper. Nonlinear elements have been added to this model to test the capability of the method concerning nonlinear rotordynamics.

\section{POLYNOMIAL CHAOS EXPANSION THEORY}

The general idea behind the PCE is to express a generic stochastic output (e.g. amplitude of displacements, frequency, etc.) as a sum of polynomials. The stochastic variable must be modelled by an appropriately chosen polynomial, facilitating the calculations of expectations [10]. The goal is to reduce the infinitedimensional random space to a finite-dimensional space that can be computed. This is accomplished by parametrizing the random space with a finite number of mutually independent random variables. Let us consider a random variable $\xi$ defined in a random space $\Omega$ of dimension $d$, with a cumulative distribution function $F_{\xi}(\xi)$ and finite moments. Considering a random variable $\xi$ and a stochastic function $y(\xi)$, the generalised polynomial chaos (truncated at the order $P$ ) expansion of $y$ is:

$$
y(\xi)=\sum_{k=0}^{P} \beta_{k} \Psi_{k}(\xi)
$$

where $\beta_{k}$ are the coefficients of the basis (or stochastic modes) that have to be calculated, and $\Psi_{k}(\xi)$ are the polynomial chaos basis functions. One considers the cumulative density function (CDF) such that $d F_{\xi}(\xi)=p(\xi) d \xi$, where $p(\xi)$ is the probability function (PDF) of the random variable $\xi$. The polynomial chaos basis functions for this distribution are the orthogonal polynomial functions satisfying:

$$
\int_{\Omega} \Psi_{n}(\xi) \Psi_{m}(\xi) p(\xi) d \xi=\gamma_{n} \delta_{m n}
$$

where $\Psi_{m}(\xi)$ are orthogonal polynomials with a weight function (the PDF $p(\xi)$ ), $\gamma_{n}$ are constants and $\delta_{m n}$ is the Kronecker delta. This establishes a relationship between the distribution of the random variable $\xi$ and the orthogonal polynomials of its PCE basis which must be selected accordingly [5, 10, 15]. Given the random space dimension $d$ and the maximal polynomial degree in the expansion $p$ (also called chaos degree), the number of 
terms in the Eqn.(1) is given by:

$$
P+1=\frac{(d+p) !}{d ! p !}
$$

The coefficients (stochastic modes), $\beta_{k}$, of the sum in Eqn.(1) can be calculated following different intrusive or non-intrusive approaches. In this paper a non-intrusive collocation method is used for this purpose.

Non-intrusive collocation method In deterministic numerical analysis, Non-Intrusive Collocation Methods (NICM) are those that require the residue of the governing equations to be zero at discrete nodes in the computational domain. These nodes are called collocation points. The NICM solves a finite number of deterministic problems obtained from the stochastic problem by replacing the random variables with deterministic values where the selected nodes are typically the nodes of a Gaussian quadrature rule (cubature rule in multidimensional space) [10]. For instance, $M$ collocation points in a one-dimensional random space are the zeros of the $M-t h$ degree polynomial of the basis.

The aim of this method is to conduct discrete projections by using the definition of orthogonality:

$$
\beta_{k}=\frac{<y(\xi), \Psi_{k}(\xi)>}{\gamma_{k}}
$$

where the symbol $\langle$,$\rangle indicates the inner product defined in$ Eqn.(2), with respect to the PDF. The above relation means that the stochastic modes can be computed by solving integrals. The problem is that the expression $y(\xi)$ is unknown at this stage and beta can therefore not be computed explicitly. In order to avoid this problem the integrals must be approximated by cubature formulas. Given a set of $M$ collocation points and the associated normalized weights (according to the PDF), after calculating $M$ deterministic solutions $y(\xi)$ the equation becomes:

$$
\beta_{k}=\frac{1}{\gamma_{k}} \sum_{i=1}^{M} y\left(\xi_{i}\right) \Psi_{k}\left(\xi_{i}\right) w_{i}
$$

The Gaussian quadrature (5) can be adapted to multivariate random spaces $(d>1)$, i.e. Gaussian cubature $[5,10]$. One of the approaches to calculate the stochastic modes in the entire space is the tensor product rule. Unfortunately, using the NICM with the tensor product rule, leads to an exponential increase in the number of collocation points with the dimension $d$. A significant reduction in the required collocation points can be achieved

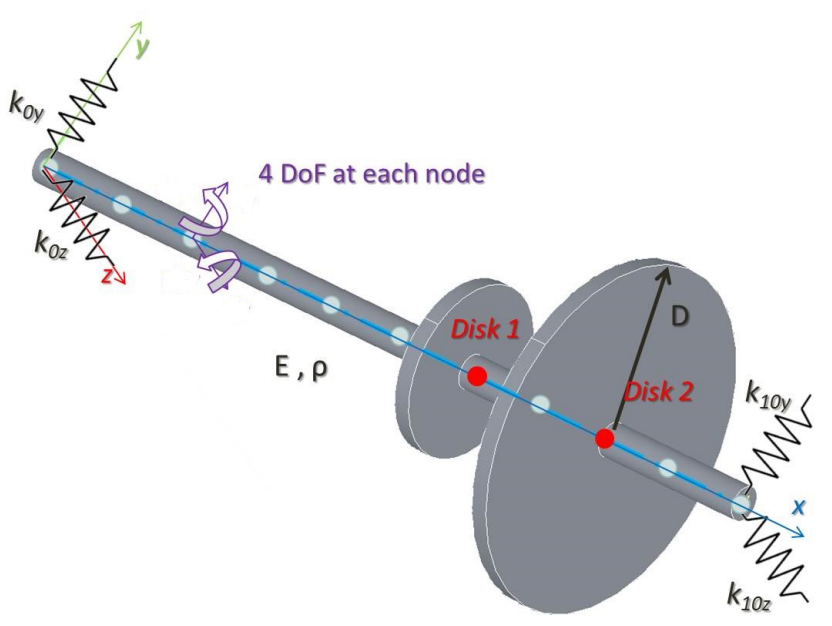

FIGURE 1. FE DISCRETIZATION OF THE ROTOR SYSTEM.

with the Smolyak sparse grids cubature. A detailed presentation of Smolyak grids construction is beyond the scope of this paper but can be found in [16]. Both collocation methods have been employed in this analysis to evaluate their effectiveness.

\section{LINEAR PROBLEM: STOCHASTIC HARMONIC BAL- ANCE}

\section{Presentation of the rotor model}

In order to validate the Polynomial Chaos Expansion (PCE) approach for the dynamic analysis of an uncertain rotor system, the model presented in figure 1 has been studied (see Reference [17]). It consists of a rotor shaft, discretized by 10 Timoshenko beam finite elements with two added rigid discs. The FE code used for this study is the open-source software Code Aster ([11]), which has a Python interface for scripting.

As shown in Fig.(1), each node holds four degrees of freedom, denoted by $[d y d z d r y d r z]^{T}$, where $d y$ and $d z$ are the translations along $y$ and $z, d r y$ and $d r z$ the correspondent rotations (expressed in the fixed frame). The system is supported at its ends by two bearings modelled by linear springs in the directions $y$ and $z$, with relative stiffness $k_{y}, k_{z}$ ). The shaft section is circular and constant with the dimensions from Tab.1. The Rayleigh viscous damping model was used for the analysis, with $\beta=0$ and $\alpha_{i}=\frac{\eta}{2 * \omega_{i}}$, with $i=1,3$ and $\omega_{1}, \omega_{3}$ being the critical speeds of the first and the third shaft mode respectively. 


\begin{tabular}{|l|r|}
\hline Parameters & Dimension \\
\hline Length of shaft & $1 \mathrm{~m}$ \\
Diameter of shaft & $0.04 \mathrm{~m}$ \\
Position of disc 1 & $0.6 \mathrm{~m}$ \\
Position of disc 2 & $0.8 \mathrm{~m}$ \\
Outer diameter of disc 1 & $0.2 \mathrm{~m}$ \\
Outer diameter of disc 2 & $0.4 \mathrm{~m}$ \\
Inner diameter of discs 1 and 2 & $0.04 \mathrm{~m}$ \\
Thickness of discs 1 and 2 & $0.02 \mathrm{~m}$ \\
Young modulus of elasticity $E$ & $2.1 E 11 \mathrm{Nm}^{2}$ \\
Shear modulus $G$ & $8.0 E 10 \mathrm{Nm}^{2}$ \\
Poisson ratio $v$ & 0.3 \\
Density $\rho$ & $7800 \mathrm{kgm}^{-3}$ \\
Unbalance mass $m_{e}$ & $0.05 \mathrm{~g}$ \\
Unbalance mass initial angle $\phi_{e}$ & 0 \\
Eccentricity of the unbalance mass $d$ & $0.02 \mathrm{~m}$ \\
Stiffness & $1 E 6$ \\
Damping factor $\eta$ & 0.03 \\
\hline
\end{tabular}

TABLE 1. ROTOR MODEL PARAMETERS.

\section{Matrix definition}

The general linear equations of motion of the shaft can be written in the following form:

$$
\mathbf{M}_{s} \ddot{\mathbf{x}}(t)+\left(\mathbf{C}_{s}+\omega \mathbf{G}_{s}\right) \dot{\mathbf{x}}(t)+\mathbf{K}_{s} \mathbf{x}(t)=\mathbf{f}_{s}(t)
$$

where $\mathbf{M}_{s}$ and $\mathbf{G}_{s}$ are the mass and gyroscopic matrices of the shaft, $\mathbf{K}_{s}$ and $\mathbf{C}_{s}$ the elementary stiffness and damping matrices, while $\omega$ is its rotational speed. The vector $\mathbf{f}_{s}$ defines the forces applied on the shaft, due to an eccentric mass distributed along the entire shaft length (differently from [17]). Unbalance rotor is one of the more common issues in rotating systems. Fig.(2) shows a cross-section of the shaft, with the unbalance mass $m_{e}$ and its initial angle $\phi$. The resulting unbalance force $\mathbf{f}_{s}$ is given by:

$$
\mathbf{f}_{s}=m_{e} d_{e} \omega^{2}[\cos (\omega t+\phi) \sin (\omega t+\phi) \quad 0 \quad 0]^{T}
$$

where $d_{e}$ is the eccentricity and the equivalent degrees of freedom are $[d y d z d r y d r z]^{T}$.

Regarding the discs, the rigid disc model is given by:

$$
\mathbf{M}_{d} \ddot{\mathbf{x}}(t)+\omega \mathbf{G}_{d} \dot{\mathbf{x}}(t)=\mathbf{f}_{d}(t)
$$

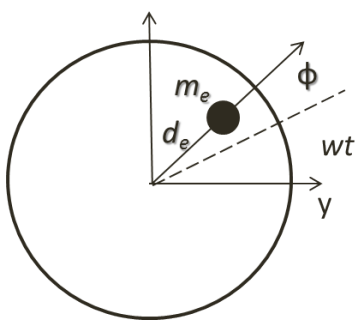

FIGURE 2. UNBALANCE FORCE ON THE SHAFT.

where $\mathbf{M}_{d}$ and $\mathbf{G}_{d}$ are the mass and gyroscopic matrices of the disc. Finally, discrete stiffness components are placed at both ends of the shaft. After assembling the shaft elements and the rigid discs, the equation of motion of the complete rotor system is:

$$
\mathbf{M} \ddot{\mathbf{x}}(t)+(\mathbf{C}+\omega \mathbf{G}) \dot{\mathbf{x}}(t)+\left(\mathbf{K}+\mathbf{K}_{b}\right) \mathbf{x}(t)=\mathbf{f}(t)
$$

where $\mathbf{K}_{b}$ is the stiffness matrix of the bearings. The expression of the matrices are given in the reference [17].

\section{Harmonic balance method}

A stochastic analysis will require repeated computation of the dynamic problem, and working in the frequency domain is more efficient than using time-domain methods. For this reason, the Harmonic Balance Method is adopted to solve the system from Eqn.(9). The displacements and the rotation, together with the forces, are represented as a truncated Fourier series with $n$ harmonics. In this study only the first harmonic is retained, i.e. the Fourier basis is simply $[\cos , \sin ]$. The response vector may then be assumed to be $\mathbf{x}(t)=\mathbf{x} e^{j \omega t}$. Substituting these expression into the equation of motion Eqn.(9) and balancing the harmonic terms, a solution projected into the orthogonal Fourier space can be obtained, i.e. the components $[\cos , \sin ]$ for each degree of freedom. The number of unknowns is 88, as the system from Eqn.(9) has 44 DOFs (11 nodes with 4 dofs each). The deterministic response of the rotor is then obtained by solving the linear system $\mathbf{A X}=\mathbf{F}$ where:

$$
\mathbf{A}(\omega)=-\omega^{2} \mathbf{M}+j \omega(\mathbf{C}+\omega \mathbf{G})+\mathbf{K}+\mathbf{K}_{b} .
$$

\section{Model validation: FRF and Campbell Diagram}

Before the deterministic model can be used in a study with uncertainties, it is necessary to validate it. For this purpose the Frequency response Function $(F R F)$ and the Campbell Diagram will be computed. 
For the $(F R F)$, the Amplitude is calculated at every $H B M$ input frequency as:

$$
A_{i}(\omega)=\sqrt{x_{i_{c o s}}(\omega)^{2}+x_{i_{s i n}}(\omega)^{2}}
$$

where $i=0, \ldots, 88$ spans the degrees of freedom in the system, while $x_{i_{c o s}}$ and $x_{i_{s i n}}$ are the solution of Eqn.(9) projected onto the Fourier basis previously defined.

Another widely used plot in rotor dynamics is the Campbell diagram, that represents the evolution of the critical speeds, corresponding to the natural frequencies, versus different operating speeds. This is possible by calculating the eigenvalues of the problem:

$$
\left[-\omega^{2} \mathbf{M}+j \omega \mathbf{G}+\mathbf{K}\right] \mathbf{A}=0
$$

assuming the solution $\mathbf{X}=\mathbf{A} e^{j \omega t}$. Only the first three modes will be considered in this study.

\section{Formulation of the linear stochastic system}

In order to formulate a stochastic system, the first step is to identify the uncertain parameters. These are treated as independent random variables (for example using the Karhunen-Loeve Expansion $K L E$, see [10]). This study will consider the following configurations (see Fig.(3)) with a maximum of two uncertain variables $(N=2)$ :

Case A : $N=2$, Normal distributions $\mathscr{N}(\mu, \sigma)$ with the mean $\mu$ and the standard deviation $\sigma=0.05 * \mu$ for the Young modulus and the spring stiffness $E, K_{y}$ (not bounded in $\Omega$ ). The metamodel is created by projecting the model into a basis of Hermite polynomials (optimal polynomials for Normal distributions);

Case B : $N=1$, Uniform distribution $\mathscr{U}(a, b)$, where $a=\mu-$ $0.05 * \mu$ and $b=\mu+0.05 * \mu$ for the variable $K_{y}$ (bounded distribution in $\Omega$ ). The metamodel is created by projecting the model into a basis of Legendre polynomials (optimal polynomials for uniform distributions);

Case C : $N=1$, Beta distribution $\mathscr{B}(r, t, a, b)$, where $r=2.5, t=$ 4 define the shape of the distribution and $a=\mu-0.07 *$ $\mu$ and $b=\mu+0.03 * \mu$ the spread of the bearing stiffness $K_{y}$. The metamodel is created by projecting the model into a basis of Jacobi polynomials (optimal polynomials for Beta distributions);

Case D : $N=2$, Beta distribution $\mathscr{B}(r, t, a, b)$, where $r=2.5, t=$ 4 define the shape of the distribution with $a=\mu-0.07 * \mu$ and $b=\mu+0.03 * \mu$ for the the bearing stiffness $K_{y}$ and

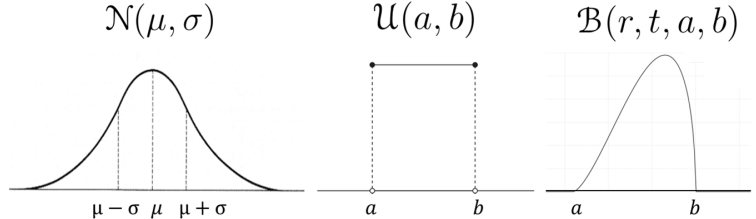

FIGURE 3. INPUT DISTRIBUTIONS FOR CASE A, B AND C.

a Uniform distribution $\mathscr{U}(a, b)$, where $a=\mu-0.05 * \mu$ and $b=\mu+0.05 * \mu$ for the density $\rho$. The metamodel is created by projecting the model into a basis of Legendre and Jacobi polynomials.

The standard deviations in these cases have been chosen arbitrarily but in the manner that they permit to validate the method. To achieve this goal they have been chosen enough large to spread the input parameter range.

Once the random input vectors have been defined, the chosen stochastic analysis can be carried out. To prove the accuracy of the suggested PCE approach, its results will be compared to the results of a Monte Carlo Sampling simulation.

\section{NON-LINEAR PROBLEM: STOCHASTIC ASYMPTOTIC NUMERICAL METHOD}

\section{Asymptotic numerical method}

In this work the method used to solve the deterministic nonlinear problem is a combination of Harmonic Balance method (HBM) and Asymptotic Numerical Method (ANM). The later technique has been proposed by Damil and Potier-Ferry [18] and improved by Cochelin [13] for calculating the post-buckling branches of plates and shells. The technique was then coupled to HBM by Cochelin and Vergez [19]. Because of the analytical representation of the solution branch in the ANM, some robust path-following technique can be used, instead of the classical predictor-corrector method [20], to get a continuous response branch.

If some localized non-linearities are added to the rotor system, the Eqn.(9) becomes:

$$
\mathbf{M} \ddot{\mathbf{x}}(t)+(\mathbf{C}+\omega \mathbf{G}) \dot{\mathbf{x}}(t)+\left(\mathbf{K}+\mathbf{K}_{b}\right) \mathbf{x}(t)=\mathbf{f}(t)+\mathbf{F}_{\mathbf{n l}}(t, \mathbf{x}(t))
$$

where $\mathbf{F}_{\mathbf{n l}}(t, \mathbf{x}(t))$ stands for the vector of non-linear forces. The first step for the use of ANM is to reduce the system in Eqn.(13) to a first-order ordinary derivative equations (ODE) system by introducing a variable $(\mathbf{v}(t))$ representing the velocity.

In order to apply the ANM for the calculation of non-linear responses, one needs to have a continuous system. For this pur- 
pose it is necessary to write each equation from (13) as the sum of constant, linear, and quadratic terms. The conversion of different kinds of non-linearity to quadratic form can be found in the literature: cubic [21], contact [22, 23, 24], friction [23, 24] and vibro-impact [23]. Using the quadratic form the system of equations can be rewritten:

$$
m(\dot{\mathbf{S}})=c+l(\mathbf{S})+q(\mathbf{S}, \mathbf{S})
$$

where $c$ is a constant vector $m$ and $l$ is a linear function and $q$ is a quadratic (bilinear) function. $\mathbf{S}$ is the vector containing all the unknowns. It should be noted that the gyroscopic effect alone $(\omega \mathbf{G} \dot{\mathbf{x}}(t))$ in Eqn.(13) produces a quadratic term versus the frequency $\omega$ without the presence of any other non-linearity in the system.

The next step is the application of the harmonic balance method to the equation 14 , which leads to the final form of the system:

$$
R(U)=L_{0}+L(U)+Q(U, U)=\mathbf{0}
$$

where $U$ is a vector containing all the Fourier coefficients for all the DOFs and the excitation frequency, and $L_{0}, L(U), Q(U, U)$ are respectively a constant, a linear, and a quadratic map. With the system in this form, the asymptotic numerical method can be applied.

The first step of the ANM is to find a solution of the non-linear system in Eqn.(15) by using a corrector method (e.g. Newton's method). After calculating the first solution, the goal is to track the evolution of the system behaviour while one of the parameters ( $\omega$ in our case) varies.

In order to find the next solutions, the unknown vector $U$ is expanded to a Taylor finite series (with order $T$ usually between 10 and 20), starting from the first solution, $U_{0}$, which has been found with an iterative process.

$$
U(a)=U_{0}+a U_{1}(a)+a^{2} U_{2}(a)+\ldots+a^{T} U_{T}(a)
$$

where $a$ is the step length that has to be calculated and $U_{1}, U_{2}, \ldots, U_{T}$ are the terms of the Taylor series. Since $R(U)$ is a quadratic operator, the calculation of these terms is simplified (as shown by Cochelin [13]). The derivatives of $U$ (used in equation (16)) can be calculated by solving a set of linear systems with the same stiffness matrix (i.e. the Jacobian matrix, see Cochelin [13] for more details).

The path parameter $a$ is defined as an arc length measure, i.e. it is identified as the orthogonal projection of the unknown vector increment $U(a)-U_{0}$ on the tangent vector $U_{1}$. The definition ensures that in a region of low curvature, the arc-length can be great due to a small variation in the amplitude, while in regions of high curvature the arc-length is reduced to minimise the number of correction steps, making the method very efficient in comparison to classical predictor-corrector method. A simple criterion to adapt the arc-length [13] is to require that the difference between two consecutive order solutions remains smaller than a tolerance $(\varepsilon)$ :

$$
\frac{\left\|a^{t} U_{t}\right\|}{\left\|a U_{1}+\ldots+a^{t} U_{t}\right\|} \approx \frac{\left\|a^{t} U_{t}\right\|}{\left\|a U_{1}\right\|} \leq \varepsilon \Rightarrow a \approx\left(\varepsilon \frac{\left\|U_{1}\right\|}{\left\|U_{t}\right\|}\right)^{\frac{1}{t-1}}
$$

\section{Stochastic formulation of the problem}

The non-intrusive collocation method is used to calculate the stochastic response.

In order to be able to apply the method to heavily nonlinear systems with turning points a special technique has been developed where the deterministic parameter used in the calculation of the stochastic modes is not the frequency which is stochastic in this case, but the arc length ratio. In this way convergence at the returning points can be ensured and the Gibbs phenomenon [10], which can appear and affect the stochastic response by introducing spurious oscillations, can be avoided. After the calculation of the deterministic solution in $N$ collocation points, the stochastic modes are calculated by integrating the scalar product either via Gaussian quadrature or Smolyak cubature (for the multivariate random spaces):

$$
\begin{array}{r}
\left(\begin{array}{c}
<\Psi_{0}(\xi), \Psi_{0}(\xi)>\beta_{0} \\
\vdots \\
<\Psi_{P}(\xi), \Psi_{P}(\xi)>\beta_{P}
\end{array}\right)=\left[\begin{array}{ccc}
\Psi_{0}\left(\xi_{1}\right) & \ldots & \Psi_{0}\left(\xi_{N}\right) \\
\vdots & \vdots & \vdots \\
\Psi_{P}\left(\xi_{1}\right) & \ldots & \Psi_{P}\left(\xi_{N}\right)
\end{array}\right] * \\
\left(\begin{array}{c}
y\left(\xi_{1}, \frac{a_{1}}{A_{1}}\right) w_{1} \\
\vdots \\
y\left(\xi_{N}, \frac{a_{N}}{A_{N}}\right) w_{N}
\end{array}\right)
\end{array}
$$

where $\frac{a_{i}}{A_{i}}$ is the arc length ratio and $w_{i}$ is the weight of the quadrature (or cubature) used for integrating the scalar product. The values of the weights depends on the number of collocation points and the cubature technique [10]. $y$ represents the deterministic solution in the collocation points.

Several different nonlinear uncertain cases have been considered. The first one (case E) is the original linear case, but by using the ANM for its solution a one non-linear term is present due to the gyroscopic effects. For case F,G,H a nonlinear cubic stiffness $\left(F_{n l}(t)=-k_{n l} y(t)^{3}\right)$ is added on the first node along y axis. 
Case $\mathbf{E}: N=2$, Normal distributions $\mathscr{N}(\mu, \sigma)$ with a mean $\mu$ and a standard deviation $\sigma=0.05 * \mu$ for Young's modulus $E$, and the bearing $K_{y}$ stiffness (not bounded in $\Omega$ ). $K_{z}=K_{y}$. The selected random variables do not introduce any asymmetries in the system. The metamodel is created by projecting the model into a basis of Hermite polynomials. The Smolyak cubature rule is used to reduce the number of collocation points;

Case $\mathbf{F}: N=2$. Similar to case E except for the nonlinear stiffness $k_{n l}=1 e 14 \mathrm{Nm}^{-3}$;

Case $\mathbf{G}: N=1$, Uniform distribution $\mathscr{U}(\mu(1-\sigma), \mu(1+\sigma))$ with a mean $\mu$ and $\sigma=0.05$ for the variable $K_{y}$ (unbounded in $\Omega$ ). The nonlinear stiffness is $k_{n l}=1 e 6 \mathrm{Nm}^{-3}$.

Case H : $N=2$, Uniform distributions $\mathscr{U}(\mu(1-\sigma), \mu(1+\sigma))$ with a mean $\mu$ and $\sigma=0.05$ for the variables $K_{y}$ and $K_{z}$. The nonlinear stiffness is $k_{n l}=1 e 14 \mathrm{Nm}^{-3}$. In this case the random variable introduces an asymmetry in the system $\left(K_{y}\right.$ can be bigger or lower than $K_{z}$ ). The effect of the random variable on the asymmetry and on the responses is analysed.

In this study only forward travelling excitation is considered.

\section{NUMERICAL STUDIES}

\section{Linear model}

This section presents the results of the Monte Carlo Simulation and the Polynomial Chaos Expansion for the linear rotor model. The obtained results in the Fig.(4)-(6) will all use a similar legend approach to make the results more comparable. Plotted in grey are all the FRFs that have been computed with the Monte Carlo Method. At each frequency, the MC distribution is characterized by the 5 and 95 percentile envelopes (white dots).

The PCE provides a stochastic metamodel for each frequency value. The output distribution has been analysed using the following statistical parameters:

Mean and standard deviation : mean $\mu$ (turquoise), standard deviation $\sigma$ for the envelopes $[\mu+1.96 \sigma, \mu-1.96 \sigma]$ (green, useful when the response is Gaussian);

Min/max envelopes : the minimum and maximum values, obtained by sampling the metamodel with an optimized algorithm in OpenTURNS (blue);

95\% envelopes : the 5 and 95 quantiles envelopes, calculated after the optimized sampling (red).

Fig.(4) shows the Deterministic (a) and the Stochastic (b) Campbell Diagrams of the rotor model for the case A. The first three modes are presented, i.e. 6 eigenvalues for each frequency. In this study only the excitation caused by an unbalance mass is
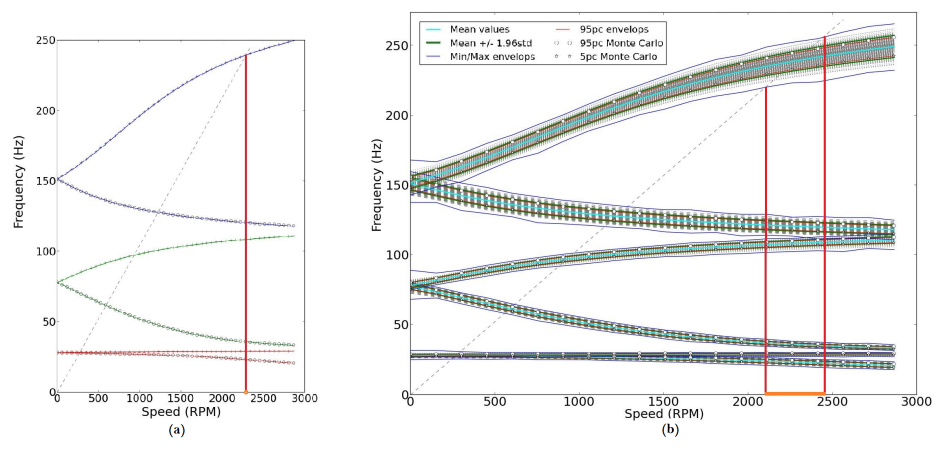

FIGURE 4. CASE A: DETERMINISTIC (a) AND STOCHASTIC (b) CAMPBELL DIAGRAMS.

considered and hence just the first engine order is traced on the Campbell Diagrams (Fig.(4) ). The size of the $M C$ sampling is 10000 and a metamodel with a chaos order equal to 2 has been used. Both methods, MCS and PCE converge to the same solution with symmetric output distributions. The $95 \%$ envelopes and the $\mu \pm 1.96 * \sigma$ of the PCE envelopes are matching and the 95 quantile points of the Monte Carlo Simulation (white points and stars) follow these envelopes as well. The minimum and maximum envelopes contain all the $M C$ samples highlighting the capability of the Min/Max metamodel to capture the dynamic response correctly for the linear case. One interesting aspect of this plot is that the intersection between the envelopes and the engine order line is no longer a single point, i.e. one singular critical speed, but a critical interval, where each rotation speed has a probability to become critical. For example, the intersection of the first engine order and the third forward mode has a critical speed interval 2100 to 2500 RPM (orange segment in Fig. 4). This means that, for an engine, the nominal speed has to be not only far enough away from the deterministic critical speed $(\sim 2300 R P M)$, but from the whole critical interval, in order to avoid possible damage to the system. The relatively large critical interval in this test case is mainly caused by the use of a relatively wide Gaussian distributions $\mathscr{N}(\mu, 0.05 \mu)$ of the Young's modulus $E$ and the bearing stiffness $K_{y}$, and it can be expected to be smaller in a real shaft assembly. Only a stochastic treatment of the problem will be able to highlight potential problems with the shaft design due to the critical interval.

In order to evaluate the influence of an uncertain shaft density on the Campbell diagram, Case $D$ was studied. In this case the density is uncertain; the mass and gyroscopic matrices become consequently uncertain. The applied bounded Beta and Uniform distributions led to a less "spread" output distribution when compared to Case A, making it easier to observe a variation from the deterministic case (where the mass matrix was constant). Fig.(5) shows the stochastic Campbell Diagram up to 5000RPM (a) and a zoom from 3000 to $4500 R P M(b)$. The higher speed range was 

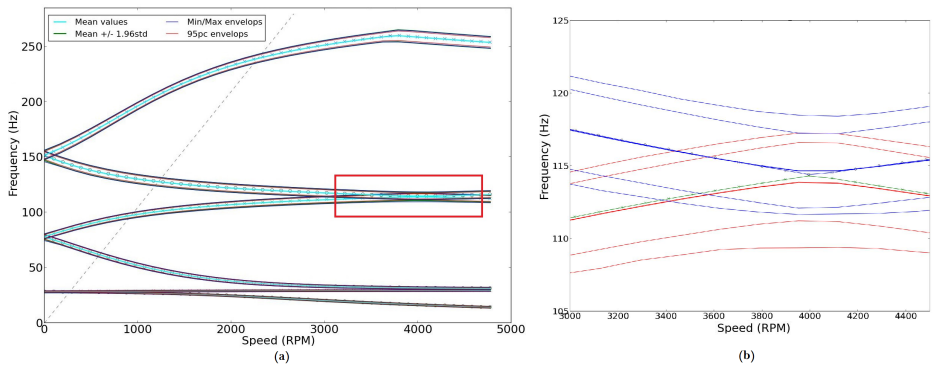

FIGURE 5. CASE D:STOCHASTIC CAMPBELL DIAGRAMS (a) AND ZOOM (b).

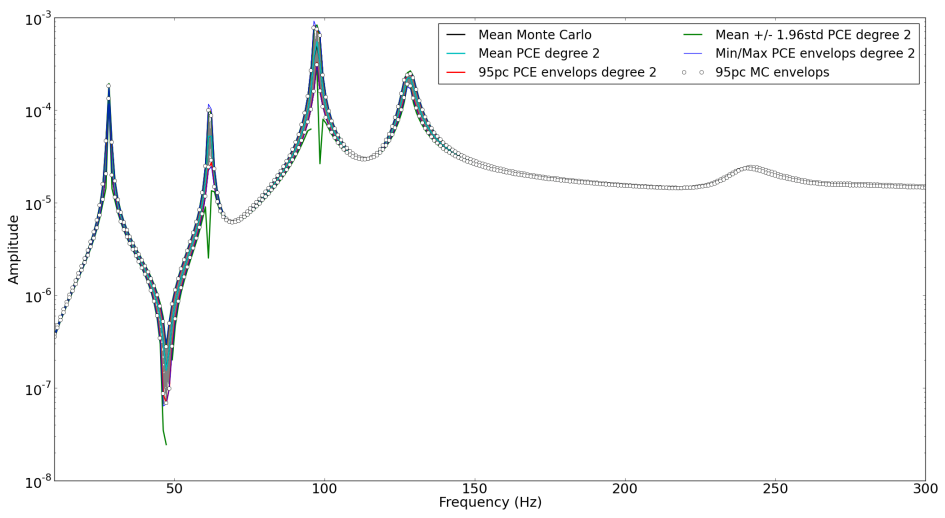

FIGURE 6. CASE B: FRF FOR DOF y DISPLACEMENT, NODE 2.

selected after observing a very close second forward mode and third backward mode in Fig. 4.

In the stochastic case, the distributions of the second forward and the third backward modes intersect in a relatively large frequency interval, compared to the deterministic case where the coincidence occurs at 4000RPM. In this interval two modes can be in resonance at the same time, which may lead to an unexpected very high amplitude response of the system. The mean values of the stochastic analysis perfectly match the deterministic case ( $x$ and $o$ on the plot), which means that a variation of $10 \%$ of the shaft density does not modify the eigenvalues of the system.

For the computation of the FRF node 2 is used, where resonance and anti-resonance are well defined. Fig.(6) shows the frequency response of case $\mathrm{B}$, for a single random parameter $K_{y}$, with a bounded Uniform distribution.

With just one uncertain input parameter, the output distribution $d y$ is relatively narrow and the $95 \%$ envelopes for both $M C S$ and $P C E$ are in good agreement. This is mainly due to the fact that the input Uniform distribution is bounded in $\Omega$.

Given the good agreement between the MCS and PCE in predict-

\begin{tabular}{|l|l|l|l|}
\hline Stochastic method & Parameter & Case A & Case B \\
\hline MCS & 10000samples & $13 h 24^{\prime}$ & $12 h 28^{\prime}$ \\
PCE metamodel & 2nddegree & $1 h 11^{\prime}$ & $15^{\prime}$ \\
PCE metamodel & 3rddegree & $3 h 16^{\prime}$ & $36^{\prime}$ \\
PCE metamodel & 5thdegree & $15 h 10^{\prime}$ & - \\
\hline
\end{tabular}

TABLE 2. SIMULATION TIMES CASE A AND B: MCS vs PCE.

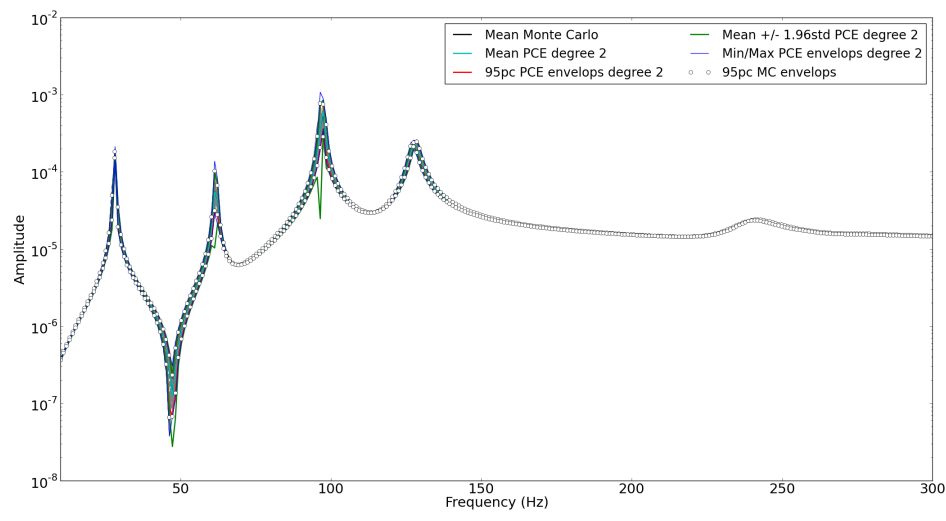

FIGURE 7. CASE C: FRF FOR DOF y DISPLACEMENT, NODE 2

ing the uncertain Campbell and FRF plots the main advantage of the PCE lies in its computational cost. Tab.2 presents CPU times for the FRF computation of case A and B. The simulation were run on a standard PC based on an Intel core i7 CPU at 3.1Ghz with 8GB RAM. Only one core was used during the simulation. As long as the number of samples remains constant, the MCS time does not change greatly between the two cases. In contrast to the MCS, the sensitivity of the PCE to the number of input parameters (higher in case A than B) and also to the metamodel degree is much stronger (the 5th degree takes as long as a MCS). All the results shown in this section were obtained with a 2nd chaos order, highlighting the accuracy of the low order model.

Fig.(7) shows the results of case $\mathrm{C}$ with a beta distribution of the bearing stiffness $K_{y}$. The output distribution is limited around the mean value, as it was for case B. The results of the PCE match the MCS results closely, even if the envelope $\mu-1.96 \mu$ shows some problems in the resonance zones caused by the Gibbs phenomenon. The conclusions are thus the same as in Case B.

\section{Non-linear model}

The effect of the uncertain parameters on the linear system response has already highlighted several issues, but even more important is the influence of the uncertainties on a nonlinear dynamics problem, since a large amount of nonlinearities can be present in turbomachinery. They are caused by the nonlinear 


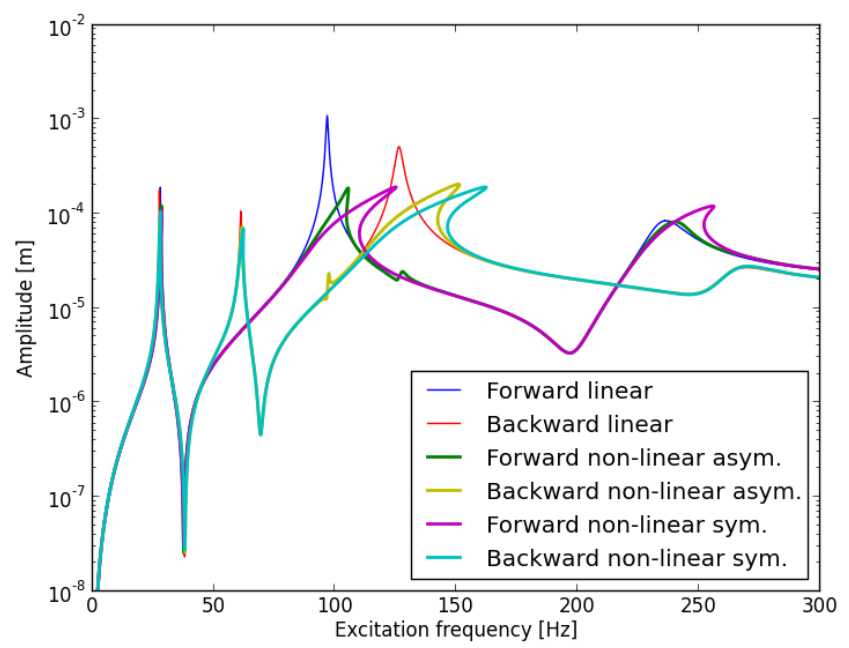

FIGURE 8. DETERMINISTIC LINEAR AND NON-LINEAR FRFs.

behavior of the components: contact in bearings, bolted joints, nonlinear dampers, impact... A important of uncertainties exist concerning the parameters of these nonlinear components. In Fig.(8) some deterministic FRFs of the displacement at node 1 for the linear and nonlinear system are shown. When the system is symmetric, the modes corresponding to the non excited direction of rotation (backward or forward) are not present, but when the system is asymmetric there is a coupling between the modes and additional peaks can be observed in the FRF. This energy transfer must be taken into account for the stochastic response since it can be strongly enhanced by uncertainty in the system.

The response of the linear case $\mathrm{E}$ that has been calculated with the stochastic ANM can be seen Fig.(9). In this case the gyroscopic effects introduce a quadratic non-linearity, but since the system is symmetric only the forward modes are excited.

The case $\mathrm{F}$ introduces a cubic stiffness along the $\mathrm{y}$-axis on node 1 , representing a potential nonlinear behaviour of the bearing. The value of the cubic stiffness has been chosen in order to observe nonlinear frequency responses with returning points. The introduction of a non-linear stiffness along one axis generates an asymmetry in the system. For this reason the backward modes are excited as well and a little peak appears around $125 \mathrm{~Hz}$ in Fig.(10). The other peaks are shifted slightly because of the hardening effect of the non-linearity, but the general stochastic distribution of the FRFs is very similar to case E.

The stochastic FRF of the case $G$ is shown in Fig.(11). In this case the asymmetry is amplified by the random distribution of one of the two bearing spring $K_{y}$, in addition to a weaker nonlinear spring. To provide a good stochastic FRF the following ap-

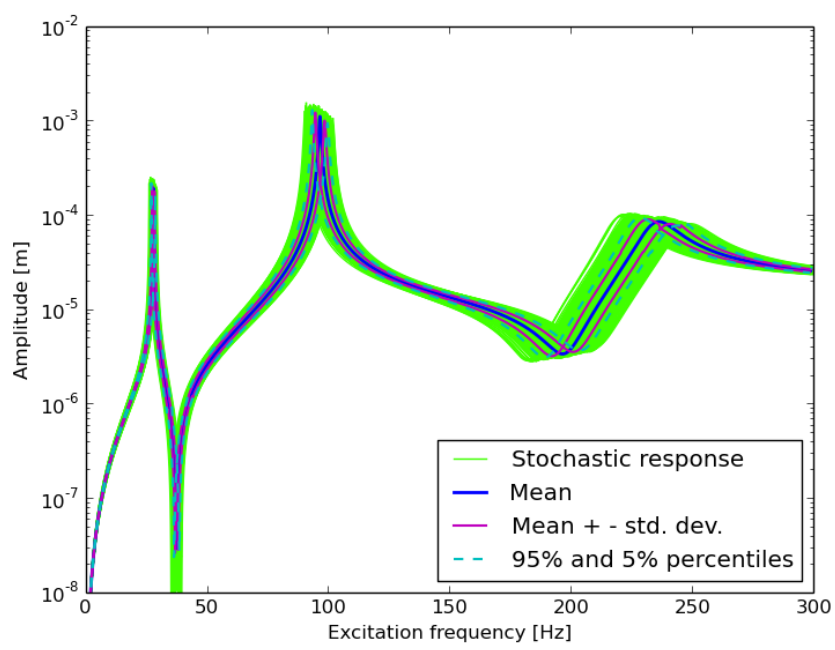

FIGURE 9. CASE E: FRF FOR DOF y DISPLACEMENT, NODE 1.

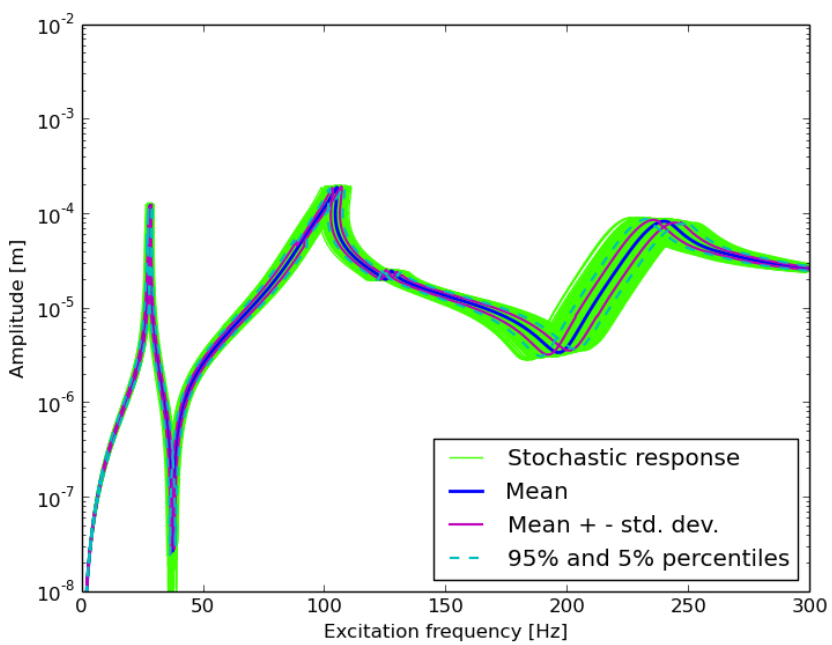

FIGURE 10. CASE F: FRF FOR DOF y DISPLACEMENT, NODE 1.

proach is being proposed; the random space has been partitioned in two subspaces $\left(K_{y}>K_{z}\right.$ and $K_{y}<K_{z}$; thus $K_{y}=\mu\left(1+\sigma u_{1}\right)$ and $K_{y}=\mu\left(1+\sigma u_{2}\right)$ with $u_{1}, u_{2}$ uniform distributions respectively $\mathscr{U}(-1,0)$ and $\mathscr{U}(0,1))$. The PCE has then been applied separately in the two subspaces and the resulting FRFs combined in Fig. (11). The backward modes are excited more strongly in this configuration due to the amplified asymmetry in the system.

For case $\mathrm{H}$ in Fig.(12) the uncertainties are introduced on the linear bearing stiffness along the $\mathrm{y}$ and $\mathrm{z}$ direction. The resulting 


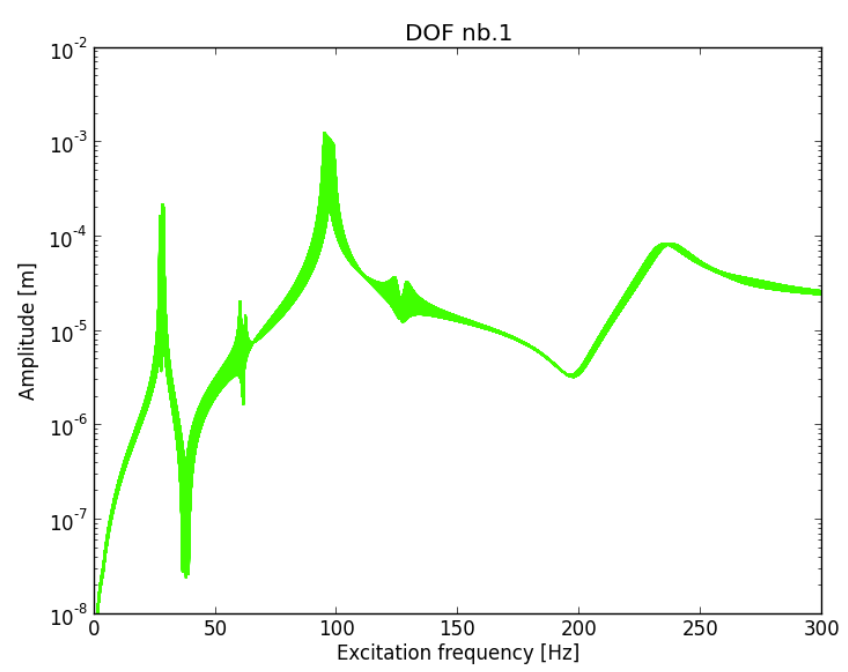

FIGURE 11. CASE G: FRF FOR DOF y DISPLACEMENT, NODE 1.

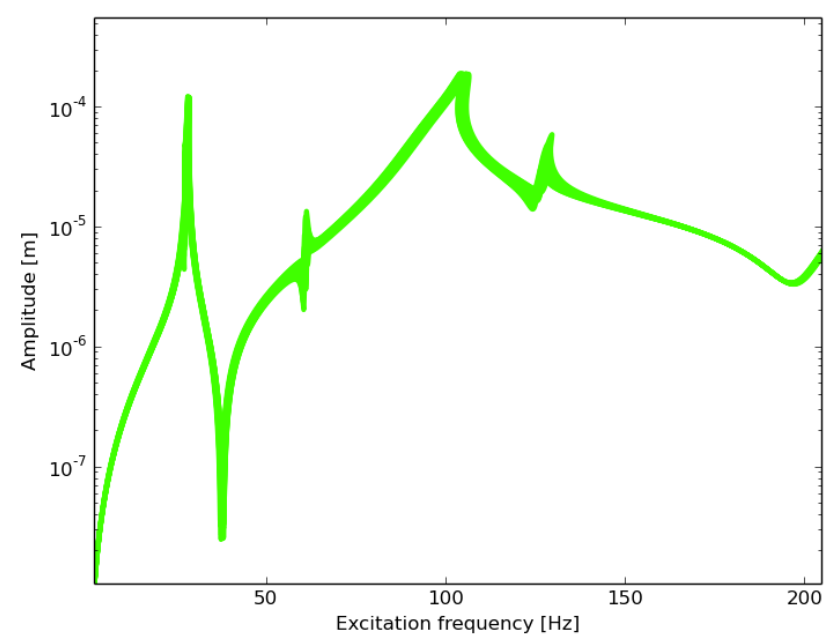

FIGURE 12. CASE H: FRF FOR DOF y DISPLACEMENT, NODE 1.

2D square random space (two uniform distributions) was divided into two triangular uniform subspaces $\left(K_{y}>K_{z}\right.$ and $\left.K_{y}<K_{z}\right)$ to reduce problems with the response calculation due to Gibbs phenomenon. An uncertainty in both directions of the linear bearing springs excites the backward modes much more than in the previous case, which is probably due to the stronger asymmetry of case $\mathrm{H}$.

All above results show, that the suggested combination of HBM, ANM, PCE and Smolyak cubature permits to reproduce the stochastic response of a nonlinear rotor, and highlights the importance of including uncertainty in a rotor dynamic analysis.

\section{CONCLUSION}

In this study, a numerical implementation of the general $P C E$ approach is proposed in order to evaluate the stochastic response of a linear and non-linear dynamic rotor model with uncertain linear input parameters. A Non-Intrusive approach has been proposed to calculate the stochastic frequency response functions and stochastic Campbell Diagrams for a simple rotor model. The obtained results show that Polynomial Chaos basis is an effective way to assess the propagation of uncertainties in a mechanical systems, offering a series of benefits over the traditional sampling methods.

The PCE shows a major advantages over the MCS for the linear rotor analysis with uncertainties, allowing the calculation of the uncertain bounds in a much more efficient way without the loss of accuracy. The stochastic Campbell Diagram obtained by the linear analysis is of great interest, since it introducing the concept of a critical speed interval, where each $\Omega$ has a probability to become critical.

The proposed non-intrusive method shows its robustness also for the non-linear dynamic analysis. Of special interest is the appearance of modes from the non-excited direction due to the breaking of the symmetry once uncertainty is applied to the linear bearings, or an additional nonlinear term is being added. These modes can influence the dynamic response of the system and lead to an additional accumulation of fatigue in areas that are normally considered safe to operate.

It could be shown, that the PCE approach can be used to improve the robustness of the dynamic design of turbomachines, also further improvements may be necessary to allow the inclusion of higher order polynomials. Future study will be carried on the effects of the uncertain nonlinearities on the stochastic response.

\section{REFERENCES}

[1] Metropolis, N., and Ulam, S., 1949. "The Monte Carlo Method". Journal of the American statistical association, 44(247), pp. 335-341.

[2] Ghanem, R. G., and Spanos, P. D., 2003. Stochastic finite elements: a spectral approach. Courier Dover Publications, Dover, New York.

[3] Xiu, D., 2010. Numerical Methods for Stochastic Computations. Princeton University Press.

[4] Wiener, N., 1938. "The Homogeneous Chaos". American Journal of Mathematics, 60(4), pp. 897-936. 
[5] Xiu, D., and Karniadakis, G. E., 2002. "The Wiener-Askey Polynomial Chaos for Stochastic Differential Equations". SIAM J. Sci. Comput., 24(2), Feb., pp. 619-644.

[6] Chantrasmi, T., Doostan, A., and Iaccarino, G., 2009. "Padé-Legendre approximants for uncertainty analysis with discontinuous response surfaces". Journal of Computational Physics, 228(19), pp. $7159-7180$.

[7] Wan, X., and Karniadakis, G. E., 2005. "An Adaptive Multi-element Generalized Polynomial Chaos Method for Stochastic Differential Equations". J. Comput. Phys., 209(2), Nov., pp. 617-642.

[8] Blatman, G., and Sudret, B., 2011. "Adaptive sparse polynomial chaos expansion based on least angle regression". J. Comput. Phys., 230(6), Mar., pp. 2345-2367.

[9] Didier, J., Sinou, J.-J., and Faverjon, B., 2013. "Nonlinear vibrations of a mechanical system with non-regular nonlinearities and uncertainties". Communications in Nonlinear Science and Numerical Simulation, 18(11), pp. $3250-$ 3270.

[10] Le Maître, O. P., and Knio, O. M., 2010. Spectral methods for uncertainty quantification : with applications to computational fluid dynamics. Series: Scientific computation. Springer, Dordrecht, Heidelberg, London.

[11] Code Aster documentation.

[12] OpenTURNS documentation.

[13] Cochelin, F. d. d., 1994. "A path-following technique via an asymptotic-numerical method". Computers \& Structures, 53(5), pp. $1181-1192$.

[14] Smolyak, S., 1963. "Quadrature and interpolation formulas for tensor products of certain classes of functions". Soviet Mathematics, Doklady, 4, pp. 240-243.

[15] Brams, C., 2013. "Spectral methods for uncertainty quantification". Bsc. thesis, Technical University of Denmark.

[16] Kaarnioja, V., 2013. "Smolyak Quadrature”. Master's thesis, University of Helsinki.

[17] Didier, J., Faverjon, B., and Sinou, J.-J., 2012. “Analyzing the dynamic response of a rotor system under uncertain parameters by Polynomial Chaos Expansion". Journal of Vibration and Control, 18(5), pp. 587-607.

[18] Damil, N., and Potier-Ferry, M., 1990. "A new method to compute perturbed bifurcations: application to the buckling of imperfect elastic structures". International Journal of Engineering Science, 28(9), pp. 943-957.

[19] Cochelin, B., and Vergez, C., 2009. "A high order purely frequency-based harmonic balance formulation for continuation of periodic solutions". Journal of Sound and Vibration, 324(12), pp. $243-262$.

[20] Allgower, E. L., and Georg, K., 2003. Introduction to numerical continuation methods, Vol. 45. SIAM.

[21] Lazarus, A., and Thomas, O., 2010. "A harmonic-based method for computing the stability of periodic solutions of dynamical systems". Comptes Rendus Mécanique, 338(9), pp. $510-517$.

[22] Moussi, E. H., Bellizzi, S., Cochelin, B., and Nistor, I., 2012. "Nonlinear normal modes of a two degree of freedom oscillator with a bilateral elastic stop". In $18^{\text {th }}$ Symposium Vibrations Chocs et Bruit.

[23] Karkar, S., Cochelin, B., and Vergez, C., 2013. "A highorder, purely frequency based harmonic balance formulation for continuation of periodic solutions: The case of nonpolynomial nonlinearities". Journal of Sound and Vibration, 332(4), pp. $968-977$.

[24] Zahrouni, H., Aggoune, W., Brunelot, J., and Potier-Ferry, M., 2004. "Asymptotic Numerical Method for strong nonlinearities". Revue Européenne des Éléments Finis, 13(12), pp. 97-118. 